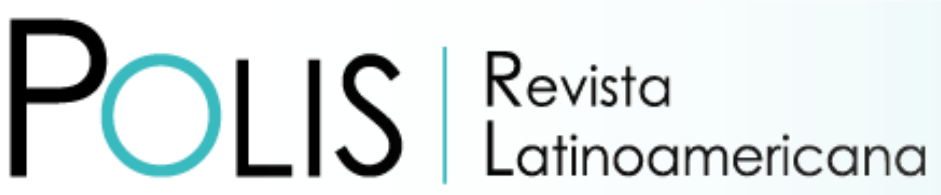

V20 | N59 | 2021

DOI: $10.32735 /$ S0718-6568/2021-N59-1588

\title{
"No nos importaba a nadie": Navegando en la búsqueda del éxito académico en Oaxaca, México
}

\author{
Mneesha Gellman \\ Emerson College, Boston, Estados Unidos \\ Email: mneesha_gellman@emerson.edu
}

Recibido: 07.10.2020 | Aceptado: 18.03.2021

\begin{abstract}
Resumen: El acceso a la educación superior y el éxito una vez allí dependen de muchas variables. En este artículo, analizo las percepciones de los principales obstáculos que enfrentan las/os estudiantes de secundaria y universitarios indígenas y de bajos ingresos en Oaxaca de Juárez, México. En combinación con un análisis estructural del contexto de Oaxaca basado en la literatura histórica comparada, utilizo la etnografía política y las entrevistas cualitativas para situar los testimonios de las/os recién graduadas/os del bachillerato y la universidad sobre sus experiencias escolares y los obstáculos que sienten que enfrentan en la búsqueda del éxito académico y profesional. Documento la matriz de obstáculos que las/os estudiantes nombran en sus trayectorias educativas, incluyendo recursos económicos, salud mental, motivación, inestabilidad política oaxaqueña, inseguridad física, climas escolares hostiles y discriminación contra los pueblos indígenas. Me baso en el estudio de caso de una organización de apoyo académico no gubernamental, el Instituto de Integración y Asesoría Comunitaria (IIAC), con sede en Oaxaca de Juárez, México.
\end{abstract}

Palabras claves: Educación; obstáculos; Oaxaca; México; intervenciones.

\section{"Nobody cared about us": Navigating the quest for academic success in Oaxaca, Mexico}

\begin{abstract}
Access to higher education, and success once there, depends on many variables. In this article, I unpack the major obstacles low income and indigenous high school and college students perceive that they face in Oaxaca de Juárez, Mexico. In combination with a structural analysis of the context of Oaxaca based in comparative historical literature, I use political ethnography and qualitative interviews to situate testimonies from recent high school and college graduates about their schooling experiences and obstacles they face in the quest for academic and professional success. I document the matrix of obstacles students name in their educational pathways, including financial resources, mental health, motivation, Oaxacan political instability, physical insecurity, hostile school climates, and discrimination against indigenous people. I draw on the case study of a non-governmental academic support organization, the Institute for Community Integration and Counseling (IIAC), based in Oaxaca de Juárez, Mexico.
\end{abstract}

Keywords: Education; obstacles; Oaxaca; México; interventions. 


\section{"Não nos importou ninguém": Navegando em busca do sucesso acadêmico em Oaxaca, México}

Resumo: O acesso ao ensino superior e o sucesso uma vez lá dependem de muitas variáveis. Neste artigo, analiso as percepções dos principais obstáculos que enfrentam os estudantes do ensino médio e superior indígenas e de baixa renda em Oaxaca de Juárez, no México. Em combinação com uma análise estrutural do contexto de Oaxaca com base na literatura histórica comparada, faço uso da etnografia política e das entrevistas qualitativas para situar os testemunhos de recém-formados no ensino médio e superior sobre suas experiências de escolaridade e os obstáculos que enfrentam na busca do sucesso acadêmico e profissional. Documento a matriz de obstáculos que os alunos nomeiam nas suas trajetórias educacionais, incluindo recursos financeiros, saúde mental, motivação, instabilidade política de Oaxaca, insegurança física, clima escolar hostil e discriminação contra os povos indígenas. Utilizo o estudo de caso de uma organização de apoio acadêmico não governamental, o Instituto de Integração e Assessoria Comunitária (IIAC), com sede em Oaxaca de Juárez, México.

Palavras-chave: Educação; obstáculos; Oaxaca; México; intervenções.

\section{Como citar este artículo:}

Gellman, M. (2021). "No nos importaba a nadie": Navegando en la búsqueda del éxito académico en Oaxaca, México. Polis Revista Latinoamericana, 20 (59), 59-78. doi: http:// dx.doi.org/10.32735/S0718-6568/2021-N59-1588

\section{Introducción}

"Antes, en la escuela, ni siquiera sabía cómo hacer las preguntas que tenía. Sin experiencia ni credenciales, nadie te toma en serio. Era como no nos importaba a nadie: a nadie le importaba si triunfábamos o fracasábamos" (Santiago Reyes, 2018).

Estefany Santiago Reyes tiene poco más de veinte años, tiene el pelo largo y negro recogido en un moño pulcro y zapatos cómodos para transitar por las calles adoquinadas de Oaxaca. Ella está sentada en una silla del salón de clases tocando periódicamente su teléfono, que se encuentra en el escritorio junto a ella. Al momento que enciendo la grabadora digital, Estefany narra cómo estaba a punto de comenzar la secundaria cuando comenzó a asistir a clases de tutoría en una organización no gubernamental (ONG) local con la esperanza de aprender habilidades informáticas.

La madre y el padre de Estefany crecieron en el sureño estado mexicano de Oaxaca, en la región occidental de la Mixteca, pero no hablaban mixteco, una de las más de 63 lenguas indígenas de México, por lo que no le transmitieron el idioma a ella. Su madre, una ama de casa y su padre, un albañil, terminaron la escuela primaria, pero no más. Antes de que ella naciera, lla madre y el padre de Estefany emigraron a las afveras de la capital del estado, Oaxaca de Juárez, en busca de más oportunidades. Ella se identifica a sí misma como indígena, y aunque nunca ha visitado los pueblos donde su madre y padre nacieron, que se encuentran en una parte de la Mixteca, plagada de conflictos civiles, continúan 
tradiciones culturales como celebraciones religiosas y un estilo de cocina que la mantiene arraigada a una identidad indígena. La jerarquía social de México ha colocado en la parte inferior a los pueblos indígenas, identificados a través de etiquetas culturales como: el idioma, el acento, la vestimenta y estereotipos a través del color de la piel, la estatura y los rasgos faciales. Las personas individuales en muchas instituciones refuerzan esta jerarquía, ya sean policías que menosprecian a las/os denunciantes indígenas o maestras/os que establecen bajas expectativas para las/os estudiantes indígenas. Cuando Estefany se enteró de un lugar al que podía ir después de la escuela para tomar clases de apoyo académico gratis, las cosas cambiaron. A alguien le importaba y ella aprendió a hacer preguntas.

Estoy sentada frente a Estefany en una habitación vacía del Amigos del Centro de Aprendizaje de Oaxaca (FOLC)', también llamado el Instituto de Integración y Asesoría Comunitaria (IIAC). FOLC es el nombre registrado de la organización en los Estados Unidos, donde recauda fondos bajo el estado de exención de impuestos 501 (c)(3), mientras que IIAC es el nombre registrado en México, lo que permite a la organización recaudar fondos allí †ambién. Utilizo IIAC a lo largo de este ensayo. Las/os estudiantes se relacionan con el IIAC de diversas formas, como mediante la recepción de tutoría académica en un rango de materias, apoyo de servicios sociales, que incluye asesoramiento sobre salud mental, y asistencia económica. Durante la pandemia de Covid-19, por ejemplo, a las/os estudiantes del IIAC se les prestaron teléfonos celulares con suficientes paquetes de datos para ayudarlas/os a llegar a la escuela en línea. No es único en el mundo en su modelo de apoyar el éxito de estudiantes marginalizadas/os, y es posible que otros casos puedan resonar con lo de IIAC. Lo que si es ejemplar es el apoyo de la comunidad internacional, que le da los recursos necesarios de cumplir su mandato.

Estefany y yo nos encontramos detrás de una fachada de hormigón en la calle Murgía, en el centro de Oaxaca de Juárez, con un patio que desemboca en varias aulas. Los murmullos de tutoría de matemáticas hacen eco en la habitación de al lado, junto con una buena cantidad de risitas y chismes. Un tutor guía a las/os estudiantes de regreso al tema en cuestión. La habilidad matemática mejorada no puede deshacer la variedad de desafíos que enfrentan las/os estudiantes de IIAC, pero las sesiones de tutoría son una parte de la prestación de servicios de múltiples frentes que intenta proporcionar herramientas para superar una variedad de obstáculos que enfrentan las/os jóvenes oaxaqueñas/os de entornos marginados. Exploro las percepciones de estudiantes y egresadas/os sobre los obstáculos para el éxito educativo en las secciones siguientes, primero mostrando el marco y los métodos de investigación, y luego presentando los obstáculos al éxito de las/os estudiantes subrayados por las/os propios estudiantes y educadoras/es.

1 FOLC, en Inglés, significa Friends of the Oaxaca Learning Center. 


\section{Marco y métodos de investigación}

En este artículo documento las reflexiones sobre los principales obstáculos que enfrentan las/os estudiantes de secundaria y universitarias/os indígenas y de bajos ingresos en Oaxaca. Pregunto qué sistemas de apoyo están disponibles para ayudar a superar los obstáculos que enfrentan y hago recomendaciones sobre cómo se podrían ampliar dichos apoyos. Basándome en la etnografía política y entrevistas cualitativas con una variedad de jóvenes y contextos escolares, centro las voces de las/os estudiantes de secundaria y universidad, junto con los recién graduadas/os de IIAC, para resaltar sus experiencias educativas. Sostengo que el éxito de las/os estudiantes se basa en muchas variables, incluida la disponibilidad de recursos económicos, la salud mental, la motivación, la inestabilidad política de Oaxaca, la inseguridad física, el clima escolar y la discriminación hacia las/os estudiantes indígenas.

Este artículo se centra en las experiencias cualitativas de estudiantes de secundaria y universitarias/os, principalmente de familias de bajos ingresos y que se identifican como indígenas o descendientes de indígenas, en Oaxaca. De 2018 a 2020, pasé varios meses seguidos en Oaxaca (cinco meses en 2018 y tres meses en 2020) haciendo investigaciones sobre la identidad y los derechos indígenas en relación a la educación, la migración y la participación. En 2018, me centré específicamente en estudiantes de secundaria y universitarias/os que afrontan desafíos para su éxito académico, y los datos de este artículo en particular provienen de ese período de tiempo. Si bien la mayor parte de mi investigación se basó en las escuelas secundarias públicas y la Universidad Autónoma "Benito Juárez" de Oaxaca (UABJO), comencé el trabajo de campo en IIAC porque, como muchas/os etnógrafas/os (iseamos honestos!), tuve un contacto allí.

Realicé ocho entrevistas a egresadas/os del programa de IIAC, junto con tres entrevistas a administradoras/es, y pasé varias horas observando a las/os estudiantes en el espacio común de ambos edificios, y también facilité un taller de dos horas para 18 estudiantes sobre género y sociedad como parte de una serie de seminarios obligatorios que IIAC realiza para las/os participantes del programa. También realicé otras ochenta y seis entrevistas con estudiantes y educadoras/es en dos escuelas secundarias públicas de Oaxaca y dos departamentos de la UABJO durante el mismo trabajo de campo de 2018, junto con nueve grupos focales y 186 encuestas (Gellman 2022, en progreso). Si bien este artículo se centra principalmente en IIAC como un estudio de caso de la resiliencia juvenil, me baso en este conjunto más amplio de datos para identificar temas generales sobre la política de educación para estudiantes de antecedentes marginalizadas, y como se enfrenten a obstáculos en su éxito académico. 


\section{Estudio de caso de IIAC}

Fundada en 2005 por un organizador comunitario jubilado de California y un maestro de escuela pública en Oaxaca, IIAC brinda servicios de enseñanza y aprendizaje académicos gratuitos a estudiantes de catorce a veinticuatro años. Las/os estudiantes atendidos son de familias urbanas de bajos ingresos y de pueblos indígenas rurales de Oaxaca que han migrado a Oaxaca de Juárez para continuar sus estudios (Quero Llaven, 2018). La programación de IIAC se enfoca en desarrollar la tripleta de potencial académico, personal y de liderazgo en las/os estudiantes (TOLC-IIAC, 2020). Al combinar la tutoría en materias básicas como matemáticas, ciencias e inglés, con tutoría y servicios psicológicos, IIAC pretende abordar el éxito escolar desde una perspectiva de persona integral (lbid). Una característica notable es que, aunque hay muchas/os voluntarias/os del programa de los Estados Unidos, el personal remunerado y las/os tutoras/es pertenecen a la misma clase social que las/os estudiantes y muchas/os son ex participantes en la programación de IIAC (Ibid). Hay muchos lugares en todo el mundo donde las organizaciones no gubernamentales (ONG) Ilenan las lagunas en la prestación de servicios dejadas por estados disfuncionales (Gellman 2010, p. 88-90), pero la política de ayudar está fuera del alcance de este documento y merece la pena consideración en otro lugar.

Las/os participantes son referidos a IIAC por amigos o familiares que han asistido ellos mismos, o por otras personas de la comunidad que conocen su oferta (Quero Llaven, 2018). Si bien hay muchos tipos diferentes de estudiantes que encuentran su camino hacia el IIAC, la mayoría tiene antecedentes de fracaso escolar por razones personales o financieras o han demostrado antecedentes de excelencia académica, pero necesitan ayuda adicional para lograr sus metas educativas (Ibid). De enero a mayo de 2018, IIAC atendió a 368 estudiantes, que es una cantidad típica para la organización. Si bien hubo algunos comentarios constructivos para la organización que provienen de las/os entrevistadas/os en IIAC, principalmente en torno a la necesidad de más espacio para acomodar a todas/os y el deseo de aire acondicionado, las/os ex alumnas/os estaban universalmente agradecidas/os por la intervención de IIAC en sus vidas, y todas/os pudieron articular claramente la diferencia que el apoyo del programa marcó en sus trayectorias personales. Las/os estudiantes actuales informaron una nueva claridad sobre sus trayectorias educativas y profesionales desde que participaron en los planes de estudio de IIAC, y un optimismo para superar sus obstáculos.

\section{Obstáculos para las/os estudiantes de secundaria y universitarias/os de Oaxaca}

En México, hay varios desafíos para estudiantes de bajos ingresos, con perfil indígena, y otras identidades marginalizadas (Alvarado Juárez y Reyes de la Cruz, 2017; Lopez Delgado, 2016). La paradoja entre el creciente pluralismo y la asimilación forzada persistente de las/os estudiantes indígenas es una con la que muchas/os oaxaqueñas/os viven a diario (Jiménez Naranjo, 2017). De hecho, esta tensión es parte del neoliberalismo multicultural 
en el estado, que es una manera de controlar la identidad y práctica de cultura por élites (Hale, 2002; Larson, 2018). A pesar de que la identidad indígena tiene reconocimiento legal en la educación pública, las barreras para la aceptación cultural de las/os jóvenes indígenas siguen siendo un problema (Secretaría de Educación Media Superior, 2014). Además, numerosos factores estructurales afectan la seguridad humana en formas que impactan las elecciones de identidad de jóvenes en México (Anaya-Muñoz y Frey, 2019). En combinación con el hecho de ser uno de los estados más pobres en México, Oaxaca como un todo tiene bastantes desafíos, con los obstáculos particulares de estudiantes de antecedentes marginalizadas aún más exacerbados.

El promedio nacional de años de escolarización K-12 es de 9.2, pero en Oaxaca es de 7.5, con solo el 16.1 por ciento de las/os casi tres millones de oaxaqueñas/os en edad escolar completando la escuela secundaria, y solo el 11.6 por ciento del total de estudiantes completando la universidad (TOLC-IIAC, 2020). El sistema de escuelas públicas de México está a cargo de la Secretaría de Educación Pública (SEP), aunque el Sindicato Nacional de Trabajadores de la Educación (SNTE), el sindicato más grande de México, tiene un impacto enorme en la gestión de las escuelas públicas y su personal. El SNTE es notoriamente corrupto y está profundamente arraigado en la red de políticas de patrocinio que caracteriza el entorno político de México (Chambers-Ju \& Finger, 2016; Cook, 1996). Desde maestras/ os que no se presentan, hasta irregularidades en la nómina, aulas superpobladas y huelgas anuales prolongadas que dejan a las familias de clase trabajadora sin cuidado de niñas/os, la gestión de la educación pública por parte del SNTE y la SEP es desastrosa en todo el país (Chambers-Ju \& Finger, 2016, p. 215). Sin embargo, la enseñanza sigue siendo una profesión honorable y un camino hacia la seguridad de la clase media para muchos. Dado que las/ os maestras/os de escuelas privadas no son miembros del SNTE, existen claras implicaciones de clase para las/os estudiantes que se ven afectados por los problemas del SNTE, ya que sus padres con los medios para hacerlo pueden optar por la escuela privada para evitar la gobernanza del SNTE.

Un alumno de IIAC procedente de una escuela pública de bajos ingresos, que ahora es profesor de inglés en una escuela secundaria privada de alto nivel, comenta:

"El sistema de educación publica es muy mala. Yo nunca tuvieron oportunidades que mis alumnos tienen. Si hubiera tenido más oportunidades... por ejemplo, mi maestro de educación física era muy gordo, pues no me desarrollaba en deportes, que era algo en que tenía capacidad. Y los libros gratuitos que nos mandaron el estado oculta cosas... [el sistema de] la educación pública necesita revisar las capacidades de los maestros, y reducir los números de estudiantes en los grupos." (Reyes Rivera, 2018).

Un estudiante actual de secundaria al que llamaré Iker inscrito en las tutorías extracurriculares de IIAC estuvo de acuerdo en que en las escuelas públicas "siempre hay más estudiantes que espacios. ¡Pide el gobierno por espacio! Y hay maestros que [el gobierno] no les pagan, y no les importan sus alumnos" (Anónimo, 2018d). Está claro que el éxito educativo está plagado de obstáculos, y Oaxaca se considera un estado de "abandono temprano" 
porque muy pocos estudiantes logran terminar la escuela secundaria superior (Kattan y Székely, 2014, p. 4). Los recursos económicos, la salud mental, la motivación interna, el intenso contexto político de Oaxaca, la inseguridad física y las culturas escolares de discriminación, especialmente para las/os estudiantes indígenas, son todas variables que atraviesan las/os estudiantes para lograr sus metas. Abordo cada variable por turno.

\section{Finanzas}

El obstáculo más dominante para las/os estudiantes oaxaqueños que trabajan para obtener sus credenciales de secundaria o universidad es la falta de recursos financieros, y esta variable fue la primera mencionada por todas/os las/os entrevistadas/os de IIAC. Las/os estudiantes a menudo toman decisiones educativas en función de su capacidad de pago, que incluyen todo, desde si pueden asistir a una sesión de tutoría (López Nicolás, 2018) hasta la universidad a la que asisten (Tanos Martínez, 2018). Por ejemplo, aunque como una gran institución pública la UABJO es la universidad más barata de la región, sus costos aún superan la capacidad de pago de algunas familias, incluso con ayuda financiera federal y estatal y becas escolares adicionales.

Para el sistema K-12, aunque la educación pública es técnicamente gratuita, las familias deben pagar los uniformes y todos los libros y materiales, y en algunas escuelas, se requiere una tarifa de inscripción y una tarifa de inscripción semestral. Efraín, un ex alumno de IIAC que ahora trabaja como enfermero, compartió que:

"Terminé secundaria pero no había dinero para continuar. Aunque los bachilleratos son públicos, hay que tener dinero para uniformes, materiales, todo. Pues empecé a trabajar limpiando un hotel siete días la semana, porque era menor de edad no pude trabajar en otra manera. Cuando cumplí dieciocho años, empecé el Bachillerato nocturno y trabajaba en el hotel las mañanas" (Pérez Hernández, 2018).

Las becas federales y estatales están disponibles para quienes califican, pero no siempre son suficientes. Si bien los nombres de becas del gobierno cambian con cada administración presidencial, son mas o menos parecidas en cuanto a cantidad y misión, la cual es prevenir la deserción escolar. En 2020, la Beca Benito Juárez para el Bien-Estar ofrece MXN $\$ 1,600 /$ S $\$ 75$ por mes a familias de bajos ingresos con un/a hijo/a en el bachillerato. Los costos educativos para la escuela secundaria varían ampliamente, ya que existen muchos sistemas diferentes e incluso dentro de algunos sistemas, las escuelas operan de forma independiente para establecer sus propias tarifas de inscripción. De mis propios sitios de investigación, los costos semestrales eran generalmente MX\$300-450/US\$15-25, y las familias también tenían que cubrir los costos del uniforme (el costo varía según la escuela y la cantidad de cambios de ropa que las/os estudiantes requieren), comida (la mayoría de las escuelas permiten vendedoras/es para vender el desayuno y el almuerzo en los terrenos de 
las escuelas) y el transporte (muy variable según la ubicación de la familia del estudiante). En algunos casos, también se necesita alojamiento, ya que las/os estudiantes que vienen de aldeas demasiado lejos para viajar de regreso a casa.

Algunas/os estudiantes se postulan y no reciben ayuda incluso cuando la necesitan (Tamayo, 2018). Martha, una participante de IIAC, dijo que su familia solo le dio permiso para seguir asistiendo a la escuela secundaria porque obtuvo muy buenas calificaciones y, por lo tanto, no le cobraron las cuotas de la escuela secundaria. Las becas de buenas promedias son salvavidas para estudiantes como ella. Aun así, trabajaba cuatro horas al día, seis días a la semana en una caseta de lotería, donde le pagaban MX\$1 1/US\$0.60 la hora (Tamayo, 2018). Martha espera que al terminar su educación secundaria pueda tener mejores oportunidades laborales que su madre y su padre, costurera y chofer, respectivamente, quienes interrumpieron su educación después de la escuela primaria (Ibid).

La Dra. Vilma Huerta Córdova, profesora de tiempo completo en el Departamento de Idiomas de la UABJO, y pedagoga experta en una escuela secundaria de artes públicas de élite en Oaxaca de Juárez, describe cómo ve la política de identidad para las/os estudiantes universitarios y de secundaria en Oaxaca. "Tenemos jóvenes de comunidades indígenas, que venden el terreno, venden la vaca, para poder comprar un espacio en la Universidad", señala (Huerta Córdova, 2018). La noción de vender la tierra o la vaca de la familia para poder pagar los exámenes de ingreso y las cuotas de las/os jóvenes para comenzar la universidad muestra claramente cómo los recursos financieros afectan directamente el acceso a la educación.

Con respecto al éxito educativo, durante mi participación semestral en los grupos de WhatsApp para varios cursos de la UABJO, con un promedio de cientos de mensajes por semana, vi menciones repetidas de preocupaciones financieras desde el costo de las fotocopias para las asignaciones hasta el transporte para llegar al campus. Además de estos costos diarios, muchas/os estudiantes migran a Oaxaca de Juárez desde las comunidades de origen para seguir estudiando, lo que conlleva gastos adicionales como el alquiler de una vivienda. Aunque el UABJO es la universidad más económica en Oaxaca, con becas y precios que varían tanto de Facultad a Facultad, todavía cuesta más que es viable para estudiantes que no tienen recursos económicos de familia, especialmente si no reciben suficientes becas.

\section{Salud mental}

El segundo obstáculo mencionado con más frecuencia para el éxito educativo de las/os estudiantes es su propia salud mental. Las/os estudiantes mencionaron la depresión y la ansiedad como dos factores que les impedían asistir o participar en clases, o realmente absorber y aprender cuando estaban en el aula. La adicción a las drogas o la experimentación ha sido un problema para algunas/os estudiantes, con distracciones como "ir de fiesta", que Iker cita como la causa de sus repetidos fracasos en la escuela secundaria (Anónimo, 2018d). 
Muchas/os jóvenes oaxaqueñas/os tienen familiares que han migrado a Estados Unidos, en particular padres, hermanos y tíos (Anónimo, 2018c). Para las/os estudiantes que han crecido viendo a su familia luchar para llegar a fin de mes, con las remesas que les recuerdan qué otras opciones podrían tener, la tendencia a la depresión o la ansiedad relacionada con la migración familiar es real. Si bien la demografía de quienes emigran desde Oaxaca ha cambiado a lo largo de los años (Stephen, 2007), la ausencia de familiares que han migrado continúa jugando un papel en la salud mental de quienes se han quedado atrás (Sánchez Cortés, 2018).

Mientras que las/os estudiantes en Oaxaca en general reportan una ausencia de atención de salud mental disponible para ellas/os, las/os estudiantes de IIAC reportan aprecio por sus servicios de consejería, así como por los talleres que hablan del desarrollo psicosocial (Pérez Hernández, 2018). Estas intervenciones les han ayudado a aprender a comprometerse con ellas/os mismas/os y sus comunidades de maneras más saludables, incluyendo la ruptura del estigma en México que mantiene silenciados los problemas de salud mental.

\section{Motivación}

El tercer obstáculo más frecuente para el éxito educativo es la motivación personal de las/ os estudiantes para realizar el trabajo escolar. Muchas/os estudiantes señalaron disfrutar y participar en el aula durante las clases presenciales, pero no estaban motivadas/os para completar o entregar la tarea o las asignaciones fuera de clase. Las/os estudiantes que han llegado a IIAC son conscientes de las posibles consecuencias de la falta de motivación, y muchos ya han sufrido fallas académicas que esperan que IIAC las/os ayude a rectificar.

La falta de motivación se ve agravada por numerosos factores. Las situaciones económicas que requieren que las/os estudiantes trabajen, los problemas de salud mental arraigados en el trauma familiar, incluyendo la separación debido a la migración, la violencia de pandillas o carteles en sus comunidades de origen, y un inadecuado apoyo familiar para estudiar, socavan el éxito educativo. La mayoría de las/os estudiantes de IIAC provienen de hogares con niveles muy bajos de educación y son las/os primera/os en sus familias en obtener títulos de escuela secundaria o universitario. Efraín, el enfermero, me dijo "mis hermanos mayores no estudiaran después de la primaria. Mi hermano pequeño es militar. Mi mamá es indígena de la Sierra Norte y habla zapoteco, pero mi papá es de la ciudad y no quiso hablar zapoteco. Los dos estudiaron hasta la primaria" (Pérez Hernández, 2018). Las/os estudiantes son muy astutas/os al vincular su propio estrés mental con la educación sobre el hecho de que no tienen familiares que les guíen en el proceso, o incluso que comprendan su valor.

La ex alumna de IIAC Anel, una estudiante de comunicaciones que recibió una beca para una universidad privada de IIAC, describió cómo: 
"Mi papá solo estudió hasta sexto de primaria, y no la terminó. Mi mama solo estudió hasta segundo de secundaria porque los maestros eran malos y nunca le gustó la escuela. Mi hermana mayor no terminó la prepa, decidió casarse antes. Y mi hermano también solo estudió la secundaria. Mi hermanita que ahora está en segundo año de la secundaria si quiere cumplir como yo" (Sánchez Cortés, 2018).

Las actividades educativas de Anel se convierten no solo en un éxito para ella, sino en una intervención en un ciclo familiar, como lo ve su hermana menor comprometida con sus estudios, tal vez con el ejemplo de Anel para demostrarle que se puede hacer. La interrupción de los patrones familiares no es poca cosa. Anel relata:

\footnotetext{
"Siempre veía como era en la casa de mi familia, mi papá es muy machista. Entonces yo decía, yo no quiero vivir esta vida, yo no quiero ser una mujer que va estar en casa y yo tenía ese deseo de querer salir y de superarme. Muchas de las amigas y niñas de mi comunidad [en el Ismo] se casan como a los 15 y 16 años. Y yo las veía y decía que yo no quiero eso para mi vida y entonces ahí, si he realizado cursos o talleres que la prepa me proporcionara, yo salí a la ciudad" (Ibid).
}

Aunque Anel comenta cómo ver a su madre sufrir bajo el comportamiento machista de su padre la llevó a trabajar aún más duro por una educación para tener un destino diferente, también describe un importante cambio intergeneracional. A medida que Anel recuperó el conocimiento y un sentido más fuerte de sí misma y propósito de sus estudios y el tiempo en IIAC, su padre comenzó a ver su educación como importante para elevar a la próxima generación, y le daría permiso continuo para asistir, a pesar del largo trayecto desde su pueblo (Sánchez Cortés, 2018). De esta manera, la motivación está relacionada a bajos niveles de educación formal de las madres y los padres y la falta de comprensión relacionada a los padres, sobre las expectativas educativas, pero la motivación también puede trascender esta limitación e inspirar a otros a percibir la educación como una oportunidad.

\section{Política oaxaqueña}

La vida estudiantil en Oaxaca se desarrolla en el contexto de un estado altamente politizado. La UABJO, como muchas otras escuelas públicas de Oaxaca, por ejemplo, es sede de huelgas laborales que cierran la escuela durante días, semanas y meses a la vez. Por décadas, y especialmente desde hace las manifestaciones de 2006 (Stephen 2013), la política domina mucho espacio logístico en la vida de oaxaqueñas/os. En cada uno de mis viajes a Oaxaca en 2008, 2012, 2018 y 2020, los sindicatos y sub-sindicatos de maestras/os, personal y otras/os trabajadoras/es afiliadas/os a la universidad, llevaron a cabo plantones, bloqueos o huelgas, llevando a la UABJO a interrumpir y cerrar las puertas principales del campus para que nadie pudiera pasar.

Los apoderamientos y otros cierres políticos de la UABJO debido a protestas del personal o de la facultad fueron citados como un obstáculo importante para el aprendizaje de las/ os estudiantes. En el nivel K-12, las/os maestras/os realizan sus huelgas anuales en torno a las negociaciones de contratos cada mayo y junio, y tales huelgas pueden durar varias 
semanas o incluso algunos meses, durante los cuales no hay instrucción (o cuidado de niñas/os) para las/os escolares. Estas protestas, por un lado, muestran a una población que ha tomado el problema de la acción colectiva en sus propias manos y está dispuesta a actuar en nombre de las demandas de las/os educadoras/es y el personal. Por otro lado, las/os estudiantes de la UABJO con las/os que hablé, estiman que generalmente pierden entre 2 y 4 semanas de clases por semestre debido a cierres por motivos políticos.

La política volátil de Oaxaca les cuesta a las/os estudiantes tiempo y dinero. Cuando el autobús o el taxi compartido de un/a estudiante llega a un bloqueo, tienen que bajar, cruzar el bloqueo bajo su propio riesgo y luego pagar nuevamente por un viaje al otro lado para finalmente llevarlos a su destino. Este gasto de transporte duplicado, así como el peligro físico que implica el cruce del bloqueo, hace que el contexto político de Oaxaca sea una carga real, como la sienten las/os estudiantes de la UABJO que no están relacionados personalmente con las protestas. Las pocas excepciones fueron las/os estudiantes que tenían maestras/os como madres o padres u otros familiares cercanos y habían discutido los bloqueos con sus ellos.

En IIAC, la mayoría de las/os estudiantes no se reconocen estar comprometidos o informados sobre la política a nivel estatal o los problemas urbanos, y ninguna/o de las/os alumnas/ os tenían madres o padres que fueran maestras/os. Los datos sugieren que las/os estudiantes envejecen hacia la conciencia política, ya que las/os ex alumnas/os del programa que tenían entre 18 y 28 años mencionaron el disgusto por la corrupción, en lugar del desinterés - la apatía como la razón de su falta de atención a la política. Un miembro del personal de TOLC-IIAS describió la variedad de tácticas de compra de votos que vio en su comunidad de origen Mixteca cerca de Pinotepa Nacional, donde la coerción social ("su familia siempre ha votado de esta manera, así que prométame que usted también lo hará") o la compra descarada ("Aquí hay 200-300 pesos, hay que votar por fulano de tal) pasa en todos los ciclos (Sánchez Peña, 2018). Está estudiando matemáticas teóricas y no quiere tener nada que ver con la política.

En contraste, las/os estudiantes que estaban en el rango de edad de catorce a diecisiete años, actualmente inscritos en programas, informaron falta de interés o irritación por los manifestantes que pedían dinero como la razón por la que no seguían la política (Anónimo, 2018d). Es lógico que las/os jóvenes puedan entrar en la política a medida que avanzan hacia la edad adulta. Sin embargo, una gran mayoría de estudiantes en varias escuelas no pudo citar de qué se trataban los bloqueos a pesar de cruzarlos regularmente (Anónimo, 2018b). Muchas/os estudiantes sintieron que el impacto negativo de los bloqueos supera cualquier propósito político y, por lo tanto, sienten ira en lugar de empatía hacia las/os manifestantes (Tanos Martínez, 2018). 


\section{Inseguridad física}

La inseguridad física es una parte constante de la realidad estudiantil, especialmente para las mujeres jóvenes. El alto nivel de inseguridad física de las/os estudiantes en su transporte hacia y desde la escuela, la tutoría y otras actividades extracurriculares fue un tema casi unánime y fuerte en la investigación. La mayoría de las/os estudiantes de la UABJO, IIAC y una escuela secundaria urbana informaron sentirse inseguros en algún aspecto de su viaje hacia o desde la escuela, ya sea por conductores o compañeros de transporte, o por tener que cruzar barricadas donde la violencia es frecuente. Las estudiantes mencionaron el temor de ser asaltadas o violadas en el transporte público, y varias mencionaron que ese incidente le había ocurrido a alguna conocida.

Algunas alumnas mencionaron que no querían asistir a clases o actividades nocturnas porque tenían que tomar transporte por la noche. Esto fue particularmente una preocupación para aquellas que regresaron a áreas más rurales donde tuvieron que hacer la última parte del viaje solos y a pie en áreas poco iluminadas. Una estudiante informó que una niña cercana a su edad había sido secuestrada recientemente en su área (Tamayo, 2018). Las alumnas dicen que es raro salir de casa solas (Sánchez Cortés, 2018; Tamayo, 2018). Si bien esto es culturalmente normal, también es emblemático el aumento de la inseguridad física de las mujeres jóvenes, que son blanco de violaciones, agresiones, robos y feminicidios, con México en segundo lugar después de Brasil en la tasa más alta de feminicidios en América Latina (Comisión Económica para América Latina y el Caribe, 2018).

Además, algunas/os estudiantes también han quedado traumatizados al presenciar la violencia familiar, nombrada violencia doméstica de sus compañeros hacia sus madres. Los estudiantes varones informaron que en general se sentían seguros en sus desplazamientos en términos de compañeros de viaje o conductores, pero algunos también mencionaron el miedo al robo y el asalto como una preocupación, y especialmente el asalto al cruzar barricadas políticas. Por lo tanto, el contexto político de Oaxaca y las preocupaciones por la seguridad física son fundamentales para las experiencias universitarias de las/os jóvenes y también dan forma a sus opciones curriculares.

\section{Cultura escolar}

Las/os estudiantes y ex alumnos de IIAC describieron el tiempo allí es como un respiro apartado de la cultura de intimidación de sus escuelas. Las/os alumnas/os y ex alumnas/os de IIAC citaron rápidamente ejemplos cuando pregunté acerca de las/os alumnas/os de las/ os que se burlaban en la primaria, secundaria y bachillerato, aunque los objetivos variaban de una escuela a otra. En el taller que facilité sobre masculinidad y roles de género en IIAC en abril de 2018, uno de los muchos talleres requeridos para las/os participantes del programa, entre los dieciocho participantes, una estudiante de secundaria dijo que sus amigas/os homosexuales y bisexuales no fueron molestadas/os y tenían una identidad sexual abierta, 
pero que las/os estudiantes indígenas con acentos eran más elegidas/os como blanco. De un asistente de taller que fue a una escuela secundaria diferente, se informó casi lo contrario, que las minorías sexuales fueron acosadas, pero eran aceptadas/os estudiantes con identificación indígena. Las/os ex alumnas/os de IIAC mencionaron que las burlas y el acoso eran peores en la escuela primaria y secundaria, y que para las/os que estaban en la escuela secundaria o la universidad, ya no observaban tantas conductas de acoso, pero varias/os tenían hermanas/os menores que informaron de acoso hacia ellas/os en sus propias escuelas (Anónimo, 2018d; Sánchez Cortés, 2018; Tamayo, 2018).

En el taller de roles de género, donde compartí una presentación de PowerPoint que destacaba temas relacionados con la igualdad de género en México y Estados Unidos, varias alumnas informaron que era la primera vez que pensaban en cosas como por qué solo se esperaba que las niñas de la familia ayuden con las tareas del hogar, o por qué los términos para jóvenes sexualmente activos masculinos y femeninos son tan divergentes (semental versus puta). A medida que las/os participantes pasaron a discusiones en grupos pequeños, la lista de formas en que las dinámicas de género discriminatorias se desarrollaron en las escuelas se hizo muy larga, incluidas las niñas que tenían que hacer más limpieza de la escuela y los niños eran llamados a responder preguntas. Con la indignación de un nuevo descubrimiento de la desigualdad que las alimentaba, las niñas en el taller comenzaron a hablar rápidamente entre sí sobre todas las formas en que veían la priorización de la masculinidad, con algunos de los niños asintiendo con la cabeza y otros retorciéndose un poco incómodos.

Mi taller fue solo una pequeña parte de una variedad de temas que se tratan temáticamente y son necesarios, junto con los servicios de apoyo psicosocial, para el enfoque de desarrollo integral de la persona para el éxito académico que adopta IIAC. Las/os ex alumnas/os comentaron que los talleres y los servicios de consejería fueron en retrospectiva altamente formativos para ayudarles a reconocer patrones y comportamientos problemáticos en sus familias, escuelas y comunidades, y también les brindaron herramientas para intervenir (Anónimo, 2018a; Reyes Rivera, 2018).

\section{Inclusión y exclusión indígena}

La intensa historia de México de discriminación racial y étnica y marginación de los pueblos indígenas tiene una relación directa con la equidad y el acceso a la educación. Estudiantes que se identifican a sí mismas/os como indígenas o que son identificadas/os por otras/ os como indígenas, en base a características como indicadores corporales, predominantemente el color de la piel o el español con acento, ya sea por hablar una lengua indígena como primera lengua, o porque el acento o el vocabulario desmienten orígenes rurales o indígenas, tienen más probabilidades de luchar por el éxito educativo. Las luchas por el éxito educativo son conectadas a procesos de discriminación, los cuales se basan en muchos factores estructurales y sociales discutidos anteriormente, ya que la identidad indígena es interseccional con los obstáculos que también afectan a las/os estudiantes de bajos ingresos. 
Debido a la discriminación, "La mayoría de gente en Oaxaca que si saben una lengua quiere ocultarla para que no se burlen", comenta Jerry, ex alumno de IIAC, "y cada vez es mas pobre el país" (Reyes Rivera, 2018). Jerry ve astutamente el mecanismo de autoprotección de ocultar o restar importancia al indigenismo como algo que empobrece aún más a México. Conecta su propia identidad como descendiente indígena, en lugar de indígena, con la colonización española: "Somos mestizos por parte de que fuimos conquistados" (Reyes Rivera, 2018).

IIAC está en línea con muchas instituciones mexicanas en términos de no tener un idioma o un mapa sobre cómo abordar la migración generacional y su cambio de identidad asociado para las/os estudiantes descendientes de indígenas. Los formularios de admisión estándar de IIAC preguntan a las/os jóvenes dónde nacieron y si hablan una lengua indígena, ya que el idioma ha sido el criterio principal para determinar si un/a estudiante se cuenta o no como indígena en Oaxaca y en México en general. Sin embargo, la categorización lingüística del indigenismo pasa por alto el fenómeno del cambio generacional de la expresión indígena en las generaciones posteriores de estudiantes de la herencia indígena. Por ejemplo, aproximadamente el setenta por ciento de las/os estudiantes de IIAC no están identificadas/os como indígenas según el proceso de admisión actual, aunque muchos de ellas/os tienen madres y padres de comunidades indígenas que hablan o al menos entienden una lengua indígena (Quero Llaven, 2018).

Las/os jóvenes que son descendientes de indígenas de segunda o tercera generación en Oaxaca generalmente no se sienten cómodas/os con la etiqueta de "indígena" si no hablan una lengua indígena. Para IIAC y otras instituciones como la UABJO y los sistemas de escuelas secundarias, una posible solución para expandir la identificación indígena podría ser agregar, después de la pregunta sobre la habilidad del idioma indígena para la/el solicitante, preguntas como "¿Dónde nacieron su madre y padre?" o "¿De dónde son sus abuelas/ os?" Un mayor conocimiento sobre la herencia indígena podría facilitar la creación de una categoría demográfica como "herencia indígena" o "descendiente indígena" que podría resultar útil para identificar de manera más integral las necesidades de las/os estudiantes.

\section{Interseccionalidad}

La forma en que la identidad indígena y su discriminación asociada afecta a las/os jóvenes oaxaqueñas/os es altamente variada. Iker, quien había venido recientemente a IIAC después de reprobar varias clases de la escuela secundaria varias veces, demostró esto en nuestra entrevista. Cuando le pregunté sobre la historia de la lengua indígena de su familia, dijo que su madre hablaba "zapoteco o mixteco, no se cual" (Anónimo, 2018d), aunque Iker ya había hablado de crecer con su madre, aparentemente lo suficientemente bien como para saber en qué idioma. habla y afliación étnica que tiene. La disociación del indigenismo para Iker parecía ser paralela su disociación académica en la que se retira de la sociedad como mecanismo de autoprotección, dejándolo en peligro de abandonar la escuela. 
Otra/os egresadas/os de IIAC enfrentaron el sistema de discriminación indígena y de clase de manera más directa. Anel describió cómo su identidad más rural e indígena la llevó a ser ridiculizada por compañeros de clase más ricos cuando asistió, con una beca, a la Universidad Mesoamericana privada en Oaxaca de Juárez:

"Yo tenía el acento de mi comunidad en el lenguaje y muchas veces se burlaban de mí de cómo hablaba, pues eran jóvenes que estudiaron toda la vida en escuelas privadas. Entonces sí sufría un poco esa parte y poco a poco fui conociendo nuevas palabras que aquí interesaban y las fui incorporando a mi lenguaje y también empecé yo a darme cuenta que una palabra que no existía en el vocabulario de aquí de la ciudad, la fui utilizando menos" (Sánchez Cortés, 2018).

Anel describe este ridículo como algo que, aunque a veces se minimiza como una burla socialmente aceptable, de hecho, desmiente a una sociedad discriminatoria:

\begin{abstract}
"Pienso que va muy pegada la palabra burla y discriminación. Era discriminación lo que ellos hacían, simplemente por la forma de vestir porque yo no vestía como ellos se han vestido toda la vida, con sus marcas. Yo no, pues, incluso mis tenis los llevaba rotos a la Universidad, y ellos me veían como fuera de lugar. Entonces sí, fue un poco de discriminación que poco a poco yo fui superando porque tengo un carácter fuerte, entonces me les enfrentaba, y así fue como superé esa parte, demostrándoles que lo que yo quería era estudiar y que yo podía ser mejor estudiante que ellos que venían de universidades privadas" (Ibid).
\end{abstract}

Al demostrar que era tan buena o mejor que las/os estudiantes de clase alta, Anel también cambió aspectos centrales de sí misma para integrarse mejor. Cuando nos reunimos para nuestra entrevista, vestía el uniforme de compañeros de edad mestizos educados: sandalias brillantes, jeans ajustados y una elegante camiseta sin mangas, con las uñas pintadas que no mostraban signos de su juventud pastoral. Me dijo que usualmente usa huipiles, blusas tradicionales indígenas tejidas y me pregunté si se había saltado el huipil en nuestra reunión para evitar ser estereotipada por mí como dijo que estaba en la universidad.

Las/os estudiantes oaxaqueños rara vez me desesperaban por los climas escolares hostiles. Numerosas/os estudiantes indígenas que entrevisté en el curso de mi investigación más amplia han dicho cosas como "Soy lo suficientemente fuerte para lidiar con eso", refiriéndose a las burlas en la escuela. Si bien Anel, debido a su carácter fuerte que se describe a sí misma, pudo reemplazar las burlas y demostrar que es una excelente estudiante, la clase y la discriminación étnico-racial que describe requerirán más que fuerza individual para revertir. Parte del trabajo de IIAC es desarrollar la fortaleza personal de las/os estudiantes para brillar como líderes mientras superan los antecedentes marginados. Las emociones embotelladas combinadas con la determinación personal pueden permitir que estudiantes como Anel tengan éxito a pesar de las probabilidades, pero no son soluciones estructurales.

Cuando le pregunté a Anel qué tipo de intervenciones pensaba que podrían ayudar a romper las prácticas sociales de discriminación, sugirió exposición cultural: 
"[La gente debe] conocer realmente la cultura, las tradiciones de las regiones, porque pienso que no están tan contextualizados de cómo en la sociedad hay diferentes tipos de culturas y pienso que estas personas sí necesitaban conocer, leer, que alguien les explicara que no todo es ciudad, sino que hay un mundo donde hay campo, pescas, muchas otras cosas donde quizás ellos nunca han pasado por eso. Nunca han pasado hambre, nunca han visto a un agricultor, nunca han ido a sembrar un árbol, una planta. Entonces sí hace falta mucha cultura en esa parte de que se les muestre o que realmente vean como son otros mundos dentro de aquí del estado de Oaxaca, sí hace mucha falta, porque se quedan de que Oaxaca es calles bonitas, centros culturales, centros artísticos, y no ven otra parte, que es la mejor parte del estado de Oaxaca" (Sánchez Cortés, 2018).

Sin embargo, no todas/os las/os estudiantes eran optimistas de que su generación formaría parte del cambio social. Iker respondió con sarcasmo a mi pregunta sobre qué significaba la democracia para él diciendo: "Democracia es de valores, de igualdad, pero no veo eso... solo egoísmo. Muchos de mi generación se drogan, hacen música, se suicidan. Hace unos años, jno me importa nada la política... los jóvenes son egoístas! Solo jóvenes buscando diversión... no les importa nada" (Anónimo, 2018d).

El descuido por cualquier cosa está en línea con el diagnóstico de lo que se conoce en América Latina como los Ninis, generalmente varones entre 15 y 24 años que "ni estudian ni trabajan" (De Hoyos, Popova, \& Rogers, 2016, pág.1). El peligro de una generación que no se preocupa por nada fuera de sí misma, y que ni estudia ni trabaja, está vinculado a la persistente desigualdad intergeneracional, así como al crimen y la violencia (lbid). El nihilismo en la juventud se conecta con el consumismo en un mundo globalizado, donde las/os jóvenes ven la prosperidad material fuera de su alcance y pueden recurrir a opciones ilícitas para obtenerla. Las implicaciones para la democratización con Ninis como una fuente creciente de identidad cultural para las/os jóvenes son claras.

Las/os jóvenes que abandonan la escuela antes de obtener un diploma de escuela secundaria tienen pocas opciones de empleo bien remuneradas, lo que hace que la participación en la economía ilícita sea mucho más probable. Dado que Oaxaca limita con estados donde los carteles ya se han apoderado de muchas de las operaciones del estado, incluida la provisión de seguridad, junto con su inverso, el peligro de inestabilidad democrática es real. El abandono escolar también juega un papel importante en la migración hacia los Estados Unidos, donde las/os jóvenes oaxaqueñas/os creen que podrían tener una mejor oportunidad de conseguir un trabajo remunerado. Identificar los obstáculos al éxito educativo es una pequeña intervención que hago para poder ver cómo los jóvenes de Oaxaca pueden evitar el fenómeno Nini, permanecer en la escuela y terminar sus estudios secundarios y universitarios. 


\section{Conclusión: definir el problema como un comienzo}

México enfrenta desafíos formidables para brindar una educación pública significativa para todas/os. Ante esta realidad, es inevitable que las/os estudiantes recurran a las ONG en busca de apoyo académico cuando estén disponibles, ya que la SEP y el SNTE no brindan una calidad de educación que permita la movilidad socioeconómica, particularmente para estudiantes de escasos recursos y antecedentes indígenas. Sin embargo, liberar a los estados de la responsabilidad de proporcionar una educación que facilite la movilidad real también socava una promesa central de la democracia, que todos tienen el derecho a participar por igual en los derechos y responsabilidades del estado. Para abordar verdaderamente la inequidad educativa en lugares como Oaxaca, la SEP y el SNTE tendrían que reestructurar fundamentalmente su funcionamiento. Si bien el presidente Andrés Manvel Luís Obrador incluye la innovación educativa en su plataforma, no está claro si el status quo cambiará para las/os escolares oaxaqueñas/os, particularmente en el contexto de la pandemia Covid-19.

Sin embargo, dados los obstáculos que enfrentan las/os jóvenes oaxaqueñas/os en sus trayectorias educativas, las ONG como IIAC juegan un papel crucial como intermediario. La falta de recursos económicos, la salud mental, la motivación, el ambiente político inestable de Oaxaca, el clima escolar y la discriminación hacia las/os estudiantes indígenas operan como variables que pueden socavar el éxito escolar. El tiempo en IIAC ayuda a las/os estudiantes a superar tantos obstáculos como sea posible. Las/os estudiantes de IIAC consideran que la participación en la programación es una inversión en su capacidad para completar la educación y obtener un trabajo con un salario inferior. Las/os egresadas/os testifican que esta percepción es correcta. IIAC constituyó una intervención singular en su vida educativa que permitió a las/os egresadas/os obtener metas educativas que antes estaban fuera de su alcance. Universalmente, las/os alumnas/os citan esta intervención como transformadora y como la creación de los medios para una vida con un trabajo digno y satisfactorio.

Las/os alumnos y ex alumnas/os de IIAC citan a familiares, personal de IIAC, maestras/os y amigas/os como fuentes de apoyo en sus trayectorias académicas. Sin embargo, otros señalan que las/os maestras/os y los miembros de la familia también pueden socavar su confianza en sí mismos, ya que algunas/os adultas/os no creen en la/el estudiante, sus habilidades o el mérito de la educación. La integración de la educación como un derecho humano deberá tener lugar no solo institucionalmente en lugares como Oaxaca, sino también culturalmente. Donde el racismo sistemático, las escuelas con fondos insuficientes y la política sindical han expulsado a muchas/os estudiantes indígenas y de bajos ingresos de la escuela, y a veces a la niñez, hay un amplio espacio para repensar cómo se ve la educación pública en México tanto a nivel macro como micro.

Mi contribución aquí ha sido articular los obstáculos percibidos para el éxito educativo de las/os estudiantes indígenas y de bajos ingresos en Oaxaca, con la esperanza de que tal claridad sobre los problemas permita superarlos estratégicamente. Cosas como la ayuda 
financiera, el asesoramiento sobre salud mental y el plan de estudios culturalmente relevante son solo algunas de las acciones que podrían marcar la diferencia para mantener el interés

y la participación de las/os estudiantes. Tener maestras/os y mentores que se presenten, se preocupen y reciban una remuneración suficiente por hacerlo también es una necesidad. Además, abordar las culturas de acoso y discriminación es fundamental para promover entornos de aprendizaje en los que se sienta seguro. Enfrentar el multiculturalismo neoliberal es urgente porque impacta la identidad de jóvenes.

Cada uno de estos obstáculos y las posibles soluciones requieren más investigación para resolver los problemas. Como un pequeño estudio de caso, IIAC está haciendo el trabajo que debería hacer un sistema de educación pública funcional. Hasta que la SEP y el SNTE, y otras entidades públicas sean capaz de brindar dicha educación, las ONG como IIAC están haciendo lo que pueden. Incumbe a todas/os trabajar para que los sistemas cambien de manera que presten atención a las voces de personas como IIAC egresada Estefany Santiago Reyes, que quiere estar tomada en serio.

\section{Agradecimientos}

Agradezco a Jaasiel Quero Llaven, Presidente del IIAC por conceder acceso a la investigación y a Robert Anyon por presentarme por primera vez a esta extraordinaria organización. También estoy agradecido a las/os alumnas/os y ex alumnas/os del IIAC por tomarse el tiempo para compartir sus historias. Emerson College me otorgó tiempo para investigación en la primavera de 2018, lo que me permitió realizar la mayor parte de esta recopilación de datos. Joshua Dankoff brindó comentarios sobre un borrador inicial y Donato Herrerra García ayudó con las correcciones en español y las transcripciones de entrevistas. Florencia Olivares Gonzalez me enseñó a utilizar un lenguaje inclusivo en español, un proceso fascinante que ofrece muchos comentarios sociales, ¡Gracias! Dos revisores anónimas/os y editoras especiales Jael Goldsmith-Weil y Kelly Baver ofrecieron comentarios útiles y cualquier error sigue siendo mío.

\section{Bibliografía}

Alvarado Juárez, A. M., y Reyes de la Cruz, V. G. (Eds.). (2017). La Educación en México: Escenarios y Desafíos. México: Universidad Autónoma Benito Juárez de Oaxaca y Juan Pablos Editor, S.A.

Anaya-Muñoz, A. y Frey, B. (2019). Mexico's Human Rights Crisis. Philadelphia: University of Pennsylvania Press.

Anónimo. (2018a). Entrevista con autora. \#18 México \#4IIAC. Alumnos del IIAC. Instituto de Integración y Asesoría Comunitaria, Oaxaca de Juárez, México. $05 / 03 / 18$.

Anónimo. (2018b). Entrevista con autora. \#24México \#5UABJO. Estudiante de origen mixe, $8^{\circ}$ semestre de zapoteco. Universidad Autónoma Benito Juárez de Oaxaca, Oaxaca de Juárez, México. 13/03/18.

Anónimo. (2018c). Entrevista con autora. \#25 México \#6UABJO. Estudiante de $4^{\circ}$ semestre de zapoteca y francés. Universidad Autónoma Benito Juárez de 
Oaxaca, Oaxaca de Juárez, México. 3/13/18.

Anónimo. (2018d). Entrevista con autora. \#77Mexico \#7IIAC. Estudiante de bachillerato. Instituto de Integración y Asesoría Comunitaria. Oaxaca de Juárez, México. 5/11/18.

Chambers-Ju, C, y Finger, L. (2016). Teachers' Unions in Mexico. En T. M. Moe y S. Wiborg (Eds.), The Comparative Politics of Education: Teachers Unions and Education Systems around the World (pp. 215-238). Cambridge: Cambridge University Press.

Cook, M. L. (1996). Organizing Dissent: Unions, the State, and the Democratic Teachers' Movement in Mexico. University Park, PA: Pennsylvania State University Press.

De Hoyos, R., Popova, A. y Rogers, H. (2016) Out of school and out of work: A diagnostic of ninis in Latin America. The World Bank. Recuperado de: https:// openknowledge.worldbank.org/bitstream/handle/10986/22349/K8318.pdf? sequence $=8$.

Economic Commission for Latin America and the Caribbean. (2018). Femicide or Feminicide. Gender Equality Observatory for Latin America and the Caribbean. Recuperado de https://oig.cepal.org/en/indicators/femicide-or-feminicide

Gellman, M. (2010). World views in peace building: a post-conflict reconstruction challenge in Cambodia. Development in Practice, 20(1), 85-98.

.(2022, en progreso). Culture Kids: Indigenous Resistance and Language Survival in Mexico and the United States. Manuscrito de libro en progreso.

Hale, C. R. (2002). Does Multiculturalism Menace? Governance, Cultural Rights and the Politics of Identity in Guatemala. Journal of Latin American Studies, 34(3), 485-524. doi:10.2307/3875459

Huerta Córdova, V. (2018). Entrevista con autora. \#46 México \#21UABJO. Profesora de UABJO y especialista de CEDART. Centro de Educación Artistico "Miguel Cabrera," Oaxaca de Juárez, México. 4/9/18.

Jiménez Naranjo, Y. (2017). Política pública y educación indígena: Entre la asimilación que no cesa y el pluralismo que no llega. En A. M. Alvarado Juárez \& V. G. Reyes de la Cruz (Eds.), La Educación en México: Escenarios y Desafíos (pp. 97-127). México: Universidad Autónoma Benito Juárez de Oaxaca y Juan Pablos Editor, S.A.

Kattan, R. B. y Székely, M. (2014). Dropout in Upper Secondary Education in Mexico: Patterns, Consequences and Possible Causes. World Bank Group, Education Global Practice Group, Policy Research Working Paper, 7083. Recuperado de https://openknowledge.worldbank.org/handle/10986/20633

Larson, E. D. (2018). Tradition and transition: Neoliberal multiculturalism and the containment of indigenous insurgency in southern Mexico in the 1990s. Latin American and Caribbean Ethnic Studies, 13(1), 22-46. doi: https://doi.org/10. $1080 / 17442222.2018 .1416895$

Lopez Delgado, M. (2016). Favouring new indigenous leadership: Indigenous students attending higher education in Mexico. Educational Research and Reviews, 11 (22), 2088-2096.

López Nicolas, E. (2018). Entrevista con autora. \#83Mexico \# 10TOLC IIAC. Director de Programa. Instituto de Integración y Asesoría Comunitaria. Oaxaca de Juárez, México. 5/21/18.

Pérez Hernández, E. (2018). Entrevista con autora. \#21Mexico \#5IIAC. Alumnus y enfermero. Instituto de Integración y Asesoría Comunitaria. Oaxaca de Juárez, Mexico.3/8/18.

Quero Llaven, J. (2018). Entrevista con autora. \# 1Mexico, \# 1 IIAC. IIAC Presidente. Instituto de Integración y Asesoría Comunitaria. Oaxaca de Juárez, México. $2 / 21 / 18]$.

Reyes Rivera, G. (2018). Entrevista con autora. \# 18Mexico, \#4IIAC. Instituto de Integración y Asesoría Comunitaria. Oaxaca de Juárez, México. 3/5/18.

Sánchez Cortés, A. (2018). Entrevista con autora. \# 17Mexico, \#3IIAC. Instituto de Integración y Asesoría Comunitaria. Oaxaca de Juárez, México. 3/5/18.

Sánchez Peña, A. (2018). Entrevista con autora, \#38Mexico \#6llAC. Coordinadora Social. Instituto de Integración y Asesoría Comunitaria. Oaxaca de Juárez, México. 3/21/18.

Santiago Reyes, E. (2018). Entrevista con autora. \#16Mexico \#2IIAC. Centro de 
Integración y Asesoría Comunitaria. Oaxaca de Juárez, México. 3/2/18.

Secretaría de Educación Media Superior. (2014). Plan de Estudios 2014: Modelo Educativo Integral Indígena (MEll). Colegio Superior para la Educación Integral Intercultural de Oaxaca (CSEllO). Recuperado de https://www.academia.edu/34542328/_Funciones_de_las_lenguas_en_el_Modelo_Integral_Ind\%C3\%ADgena_2015_CSEIIO

Stephen, L. (2007). Transborder Lives: Indigenous Oaxacans in Mexico, California, and Oregon. Durham y London: Duke University Press. .(2013). We Are the Face of Oaxaca: Testimony and Social Movements. Durham and London: Duke University Press.

Tamayo, M. (2018). Entrevista con autora. \#78Mexico \#8IIAC. Estudiante de bachillerato. Instituto de Integración y Asesoría Comunitaria. Oaxaca de Juárez, México. 5/13/18.

Tanos Martínez, I. (2018). Entrevista con autora. \#24Mexico, \#4UABJO. Estudiante de $4^{\circ}$ semestre Francés. Universidad Autónoma Benito Juárez de Oaxaca, Oaxaca de Juárez, México. 3/13/18.

TOLC-IIAC. (2020). About Us. Recuperado de https://www.tolc.org.mx/aboutus-290409.html:

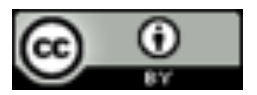

Este es un artículo de acceso abierto bajo licencia Creative Commons Reconocimiento 4.0 Internacional 